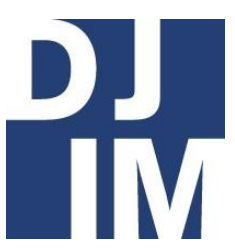

Volume 14

Spring

2018

djim.management.dal.ca |

\title{
Chasing Aces: Asexuality, Misinformation and the Challenges of Identity
}

Adrienne Colborne

School of Information Management, Dalhousie University

\begin{abstract}
Asexuality is a deeply misunderstood and little-known sexual orientation. This is partly due to misconceptions and marginalization of asexual people, and partly by a lack of information about the orientation. This paper outlines the misconceptions of the 'causes' of asexuality, namely Hypoactive Sexual Desire Disorder (HSDD), abuse, and religious abstinence. These causes are shown to be invalid due to the key element of selfidentification in determining an orientation; nevertheless, they persist in society because little is known about the nature of asexuality. The facets of the asexual orientation are then discussed: levels of sexual attraction, sexual desire, and romantic orientation, displaying the complex attempt to define asexuality, made even more difficult by a lack of sources concerning these facets. Finally, the tension between the LGBTQ+ community and asexuals is discussed in terms of the debate about including asexuals in this community, with the groups often speaking at cross-purposes. It becomes clear that being asexual requires a complex navigation of territory, and this problem is exacerbated at every step by a lack of information. It is therefore crucial that this informational gap is addressed at each of these three critical areas in order to build a more complete societal grasp of asexuality, and to create a vibrant, open community for those who identify as asexual.
\end{abstract}

The last century has seen a tremendous amount of progress in the acceptance of difference. Around the world, civil rights are slowly being granted to women, people of colour, and people in the LGBTQ+ community. There is work still to be done, but there is hope for a society which is safe for people of all races, genders, and 
sexualities. The last category, however, is still being negotiated on several levels. The LGBTQ+ community is underrepresented in media, often suffers from a public lack of understanding, and is sometimes denied access into the rest of society.

There is another group of people who are discriminated against for their sexuality, but unlike the LGBTQ+ community, they are not always granted the right of being recognized as a $g$ community. These people are asexual. Human asexuality is a relatively new concept in society (Bogaert, 2006), and as an orientation it is suppressed by a lack of information, creating difficulty for those who identify or may identify as asexual in the future. With little academic research being done into the subject, asexuality is often dismissed as a manifestation of illness, both physical and mental. This is made worse by the spread of false information as asexuality is often linked to abuse, religion, and even age. The lack of information continues within asexual literature. While sites like the Asexuality Visibility and Education Network provide information on the various aspects of asexuality, it can be overwhelming and difficult to determine which factors are the most vital, or what the line may be between 'asexuality' and sexuality. This is further complicated by having few other sources. Finally, the asexual experience is complicated by its highly contested links to the LGBTQ+ community. While some argue that asexuality is 'queer' while others argue that asexuality has no place in the LGBTQ+ community and can pose a threat to its 2 existence. It is unclear which side will emerge victorious, but the fact remains that this conflict is linked once more to the confusion and lack of information surrounding asexuality.

The state of asexuality in society is an undesirable one; fractured, misrepresented, and nearly invisible. It will take work to fix society's notions regarding asexual individuals especially in terms of distributing correct information, which will enable people to learn about this type of sexual orientation, discover if an individual is asexual, and form a strong, visible community on their own terms.

The process of defining asexuality, both in terms of a valid orientation and an identity, will be outlined to explore this issue. The connections of the asexual community and the LGBTQ+ community will then be highlighted. Each part will demonstrate the problems of information that affect these processes, but they will also provide direction as to potential solutions.

Asexuality is defined here as a sexual orientation characterized by a lack of sexual attraction (Decker, 2015). This paper uses asexual and asexuals to describe a person or people on the asexuality spectrum, and 'allosexual' to describe those who are not (Decker, 2015).

\section{Asexuality in Humans: The Road to a Definition}

Until 2004, the term 'asexual' was used almost exclusively in biology to describe the process of self-reproduction 
undergone by cells and similar life forms (Brotto \& Gorzalka, 2015). The Asexuality Visibility and Education Network (AVEN) was founded in 2002 by David Jay, but it took a study by Anthony Bogaert in 2004 to bring the word into the academic community (Brotto \& Gorzalka, 2015). In this study, Bogaert did a survey of the British population and demonstrated that about $1 \%$ of respondents experienced a lack of sexual attraction, creating a shift in perspective on the issue (Brotto \& Gorzalka, 2015).

A significant issue remained: namely, whether asexuality might be caused by mental or physical illnesses. The underlying assumption in this question is that sexual attraction is a fundamental part of being human, therefore those who do not experience it might be "manifest[ing] some underlying psychopathology" (Brotto \& Gorzalka, p. 649). Studies were duly conducted to verify whether conditions like depression or Asperger's were more common among those who reported a lack of sexual attraction; the former was found to have no significant presence, while the latter was slightly higher (Brotto \& Gorzalka, 2015).

The most commonly cited psychological ailment linked with asexuality is Hypoactive Sexual Desire Disorder (HSDD) (Brotto \& Gorzalka, 2015). The primary symptom of

1 This scale was created by the authors of the study (Brotto \& Gorzalka, 2015). The scale is not linked to AVEN, and there is a certain amount of controversy this disorder is a lack of interest in sex, so the explanation is somewhat plausible. One study discovered that there were people who identified as asexual who were suffering from HSDD (Brotto \& Gorzalka, 2015).

However, there are two main arguments against asexuality being synonymous with HSDD. When both groups of people were given the Asexuality Identification Test, or the Asexuality Identification Scale, ${ }^{1}$ those with HSDD had significantly lower scores, and rarely identified as asexual (Brotto \& Gorzalka, 2015). Secondly, the distress levels in those who self-identified as asexual about their lack of attraction was significantly lower than those diagnosed with HSDD (Brotto \& Gorzalka, 2015). This indicates that those with HSDD were more likely to report a desire to engage in sexual activity but was made difficult by their lack of attraction (Brotto \& Gorzalka, 2015). These key differences suggest quite strongly that there is a difference between the disorder and asexuality, as the effects of the disorder are felt quite negatively by those who still register on the Asexuality Identification Scale as allosexuals. This is reflected in the DSM- $V^{2}$ which excludes a diagnosis of a sexual desire disorder when an individual identifies as asexual is made (Brotto \& Gorzalka, 2015). The power of self-identification here cannot be stressed enough: it is the choice of the individual,

around the questions, which falls outside the scope of this paper.

2 The Diagnostic and Statistical Manual of Mental Disorders, fifth edition. 
upon evaluating their life experience, to identify as asexual. This is considered enough to dismiss a disorder diagnosis.

There are other factors used to disprove asexuality as some suggest a connection between sexual abuse and asexuality, especially sexual abuse in childhood (Cerankowski \& Milks, 2010). However, according to AVEN's Frequently Asked Questions page, studies have been unable to find any explicit connection (AVEN, n.d.-

a). Others argue that the lack of sexual attraction might be connected to age: that people are too young or too old to be experiencing true sexual attraction, but this again has not been proved by any scholarship (Decker, 2015). Finally, some assume that asexuals are simply celibate, but AVEN notes that celibacy is a choice concerning sexual practice, not the experience of a lack of attraction (AVEN, n.d.-b).

If these factors are removed, the remaining hypothesis is that asexuality might be its own sexual orientation. Using Bogaert's admittedly narrow definition of sexual orientation as "one's subjective sexual attraction to the sex of others," (Bogaert, 2006, p. 244), it follows that asexuals experience a lack of sexual attraction to others, are experiencing sexual attraction in the negative. As there is a lack of evidence to prove that asexuality derives from a disorder or traumatic experience, this lack of desire can be categorized as a sexual orientation.
When asexuality is seen as a sexual orientation then it can center around selfidentification; if someone is identifying their experience of this lack of attraction as asexuality, then they are asexual, regardless of sex drive. This is known as the collective identity model which allows people to claim their sexual identity weather it is asexual or allosexual (Cerankowski \& Milks, 2010). This self-identification is supported by AVEN (n.d.-b), who states that "[a]sexuality is like any other identity [...] if at any point someone finds the word asexual useful to describe themselves, we encourage them to use it for as long as it makes sense to do so" (para.8).

Allowing individuals to claim their sexuality is important, but it should be clarified that asexuality is an orientation, not something an individual can decide to 'be'(Decker, 2015); which is true of all sexual orientations. Rather, the power of selfidentification comes from an asexual person being able to use the word to describe their orientation. The choice of term may change during a person's life. For example, someone might believe they are straight but eventually clarify themselves as bisexual. However, this person does not 'become' or 'choose' bisexuality, just as asexuals do not 'choose' asexuality; their orientation has always been the same, just with a different word or evaluation. By explicitly linking asexuality to selfidentification, as well as to other identities, AVEN and the DSM-V demonstrate that asexuality is itself a valid form of identity, one that needs no outside explanation. 
However, these false assumptions about the 'causes' of asexuality continue to circulate. An underlying assumption is that everyone experiences sexual attraction, and these arguments hold power since they give both physical and psychological explanations for this lack of experience. Despite the efforts of groups like AVEN and studies like Brotto and Gorzalka's HSDD survey, these assumptions continue, making it difficult for people to self-identify as asexual. As a result of these societal perceptions an individual experiencing a lack of sexual attraction would need to have access to have access to research about asexuality to understand their sexual orientation rather than hurt or ill. From Bogaert's study is accurate across nations, there is a portion of people who may be telling the truth when they call themselves asexual. However, there may be people who lack the information to understand their own identity, and therefore the numbers of asexual people who do not recognize themselves in the incorrect portrayal of this identity.

\section{Ace Wild: The Facets of the Asexual Identity}

The ambiguity surrounding asexuality is not entirely because of misconceptions. The experience of asexuality is widely varied, and AVEN freely admits this fact. They suggest that asexuality may be better thought of as a spectrum, rather than a singular state (AVEN, n.d.-a). The asexual identity consists of three main facets: a) the level of sexual attraction, b) the level of sex drive and c) romantic orientation.

There are different levels of experiencing sexual attraction which may fall under the asexual spectrum. There are people who experience low levels of attraction who fall into the gray-asexual domain, there are people who experience no sexual attraction until they form an emotional connection who are known as demisexual, and finally there are people who have never and will never experience attraction to anyone (AVEN, n.d.-a). The lines between these experiences of sexual attraction are blurry, so it is no wonder that there is confusion regarding what 'kind' of asexual one may be, or how 'asexual' one is to fit into one of these categories. Again, AVEN welcomes people using the word while they attempt to navigate their sexuality, but the confusion remains.

While asexuality is centered around a lack of attraction, this does not necessarily preclude a lack of sexual arousal. In fact, sexual attraction as an experience is a separate entity from sexual arousal, which is itself a physiological response (Decker, 2015). Furthermore, it is also separate from sex drive, which Decker defines as "a desire to pursue sex" (2015). Consequently, there are different levels of sexual arousal and sex drive present in the asexual community. Some asexual people are completely sexrepulsed, but they are at the extreme end of the spectrum (Decker, 2015). Others are willing to have sex with a committed partner in order to please them, and 
compromise on the frequency of sex (Decker, 2015). There are even some asexuals who enjoy sexual acts but still do not feel attracted to their partner (Decker, 2015). These desires are occasionally influenced by the person's libido, but sometimes not; there are those who have a healthy sex drive but have no interest in sex or who even feel repulsed by the act (Decker, 2015). This spectrum extends to masturbation; while some asexual people engage in this behaviour, others do not (Decker, 2015). Because these aspects of sexual life are separate from sexual attraction, none of these behaviours "disqualify someone from being asexual" (Decker, 2015). On the other hand, navigating the boundaries between these definitions and deciding what level of sexual arousal and drive one experiences is challenging and would certainly provoke doubts.

Leaving aside the question of sex for a moment, asexual people also possess a romantic orientation, a separate concept of orientation used to determine a person's romantic leanings, which may be utterly unrelated to their sexual orientation (Pinto, 2014). Some asexuals want to have a romantic relationship, though how much sexual activity that relationship contains depends on the compromise made between the partners. Others, who define themselves as aromantics, are utterly

\footnotetext{
${ }^{3}$ It should be noted here that a full discussion of aromanticism falls outside the scope of this paper, particularly because aromanticism is not only

uninterested in romantic relationships, though like other humans, they still want some level of intimacy (Decker, 2015). ${ }^{3}$ Pinto argues that romantic orientation is not a spectrum because each type is distinct from each other (2014). If Pinto (2014) is accurate, asexuals may experience the same romantic orientations which allosexual people do, and their experiences may be totally distinct from another person with the same orientation.

The final 'complication' of the asexual identity is that those who experience romantic attraction may have romantic feelings for people which fall under the LGBTQ+ definition. There are heteroromantic (romantically interested in people of the opposite gender), homoromantic (same gender), bi- and polyromantic (interested in two or more genders) asexuals, and this applies to the entire spectrum (Decker, 2015). Coupling this with different levels of intimacy or desire, thus causing confusion surrounding these labels. The asexuality and queer intersectionality question will be addressed in the next section, but it is another factor which comes into play when understanding asexual identities.

With all of these factors, it should be clear that building an asexual identity is a complex affair. These factors do apply to other identities, for example: someone who identifies as heterosexual may experience

experienced by asexual people; there are people of many sexual orientations who also define themselves as aromantic. 
some mild attraction to the same sex and might be utterly uninterested in romance, while someone who identifies as homosexual may have a strong desire for romance and has a very low sex drive. In the case of asexuality, however, these spectrums and orientations are not well known or discussed. Because so many exist on a spectrum, some may feel tempted to draw a line and once that line is crossed, they can no longer consider themselves asexual.

Having a romantic orientation as well as a sexual orientation in identity issues become more complex. While sexual and romantic orientations are distinct entities, the line is blurry, especially when they coincide. There may be little distinction for someone who is homosexual and homoromantic, as opposed to bisexual homoromantic. It is entirely possible that one may become more important than the other, such as the case of romantic asexuals who are willing to engage in sex with a loved, trusted partner.

These sexual categorizations create an overwhelming amount of information to synthesize when attempting to build an identity, and tinformation on this topic is difficult to find, making it more difficult to make these decisions. Fortunately, AVEN has all of this information, but outside of its space there are few sources on asexuality. When searching the Halifax Public Libraries catalogue for 'asexuality,' there are only five different non-fiction books with asexuality as a subject (Halifax
Public Libraries, n.d.). There is only one resource which is solely about asexuality while the others discuss sexuality in general and even schizophrenia (Halifax Public Libraries). There are three fiction books which mention asexuality in their descriptions, but are not focused upon the experience of asexuality (Halifax Public Libraries). This lack of resources hinders the points of reference, thus forcing an asexual person must analyse their own experiences to compare their sexual experiences with others to gauge where they are on the spectrum. This example assumes an individual is self-aware and questioning their sexuality. However, when an individual is unsure of their sexuality it often means they are unsure how to describe their sexual identity, which may lead to multiple conversations explaining your own sexual orientation. Asexuals may have access to the information regarding asexuality, but this may result in reading material that is ambiguous and completely subjective. The ambiguity in material regarding asexuality is not necessarily a bad thing since as asexuality is a spectrum, and one can have a range of experiences. However, few reference points result in people struggling to find material that defines their experience, and may not be able to determine the 'right' criteria for being asexual, should that even exist.

\section{Adding an A(?): Asexuality and the LGBTQ+ Community}

In the previous section, the possibility of asexuality meeting the LGBTQ+ community 
was raised in the context of romantic orientations. If an asexual person is romantically attracted to people of the same sex or to multiple genders, they may be able to classify themselves as LGBTQ+. However, there is a growing movement that asexuality itself belongs in this community. This has caused a lot of debate in both the LGBTQ+ community and in the asexual community, as there are strong arguments on both sides.

Before beginning this section, it is important to clarify the vocabulary used. The LGBTQ + community covers sexualities like homosexuality and bisexuality, and genders like transgender, among several others, hence the.$+{ }^{4}$ The ' $Q$ ' stands for 'queer', which describes anyone who is not straight (Canning, 2015). This word was once a slur, and several older members of the community protest its reclamation as it is reminiscent of violence done against their community (Canning, 2015). Since the word 'queer' is contentious, the acronym LGBTQ + will be used in its place, but any citations which contain 'queer' will not be altered to respect the positions of the authors on this issue.

Those who argue that asexuality belongs in the LGBTQ+ community begin with the fact that asexuality is a minority group which often faces discrimination, much like members of the LGBTQ+ community. If the findings of Bogaert's study may be extrapolated to the rest of the world, only
$1 \%$ of the population is asexual (2006) which indicates that asexual individuals are clearly a minority. It is also true that asexuals often face discrimination, both among the general population and among other minority groups for their "unnatural" lack of attraction (Brotto \& Gorzalka, 2015). They "lack social credibility" (MacNeela \& Murphy, 2015, p. 800), largely due to the factors discussed in the first section; asexuality is "pathologized and medicalized," often denied the title of orientation (Cerankowski and Milks, 2010, p. 661). People do not talk about asexuality (MacNeela \& Murphy, 2015), and with that lack of discussion there are more instances of it being misunderstood and even mocked, creating a cycle of ignorance and repression. In a world where "great power" is attached to sexuality (Cerankowski \& Milks, 2010, p. 661), asexuals stand in defiance, but they often stand alone.

Then there are those who claim that asexuality by its nature fits in the definition of 'queer' sexuality. Cerankowski and Milks (2010) point out that asexuality challenges "perhaps the most fundamental assumption about human sexuality: that all people experience, or should experience, sexual desire" (p.650). Asexuality shows that not only is heterosexuality not compulsory, but that human sexuality itself is not universal (Gressgard, 2013). By its very existence, asexuality "decenters humans from sexuality" (Gressgard, 2013, p. 188),

4 The acronym is as follows: $\mathrm{L}$ (lesbian), G (gay), B (bisexual), $\mathrm{T}$ (transgender) and $\mathrm{Q}$ (queer) 
and makes room in the theory of sexuality for an experience of life which has nothing to do with sex. Furthermore, Miller (2015) claims that 'queer' is "inclusive of any variety of experience that transcends what has been socially and politically accepted as normative categories for gender and sexual orientation" (p.38). As asexuality challenges the sexuality narrative, it may therefore be defined as 'queer,' and thus within the LGBTQ+ community. It should also be noted that there are LGBTQ+ associations which accept asexuality as an ' $A$ ', such as Dalhousie University's DalOUT group (DalOUT, personal communication, November $\left.30^{\text {th }}, 2016\right)$. In those groups, the assumption is made that LGBTQ+ safe spaces are also safe for asexuals.

The inclusion of asexuals within the LGBTQ+ community is not the ruling idea. . In a group which is oppressed due to their sexuality, some feel that asexuality does not belong. Some argue that the marginalization of asexuals is not comparable to that of queers in a "heterosexist world"; while there are similar elements, there may not be enough overlap to truly call asexuals 'oppressed', or at least not to the degree of the LGBTQ+ community (Cerankowski \& Milks, 2010). Furthermore, since asexuality is a movement against sex, some LGBTQ+ people feel that their own movement to experience their sexuality in their own way will be threatened (Canning, 2015). This is related to the conflation of asexuality with celibacy (AVEN, n.d.-a). This has been disproven above, but if this conflation Chasing Aces spreads, it may "reinforce the idea that sexual orientation can be controlled or chosen" (Canning, 67). This idea about choosing an orientation could threaten the freedom of LGBTQ+ people who have long argued that sexual orientation is not a choice (Decker, 2015).

There is also debate within the asexual community about whether they identify as part of the LGBTQ+ community. While some asexuals see themselves as 'queer', others consider their relationships conventional and are not part of the LGBTQ+ community (Pinto, 2014, p. 332). Much like the process of identifying as asexual, this is very much about personal preference and choice (Pinto, 2014, p. 332). This may also be affected by an asexual's romantic orientation; someone who is heteroromantic may feel less connected to the LGBTQ+ community, while those who are homo-, bi-, or polyromantic may feel more 'queer' and may be more welcome in the LGBTQ+ community (Canning, 2015, p. 61).

This debate about whether asexuality fits into the LGBTQ+ community is primarily concerned with the rights of identity. Determining a group's place within another, when both groups suffer from discrimination, is an undertaking fraught with considerations of inclusion, rights, and the complex consideration of whether these groups share enough elements to band together. Both sides of the debates have compelling arguments about where asexuals should fall, and the socio-historical 
backgrounds to both are impossible to fully assess within the scope of this paper. The most basic assumption underlying both sides, however, is that 'asexuality' is 'other' than either straight or LGBTQ+. It is evident that more discussion is needed on this topic, but this cannot take place only within one group or another. By creating a dialogue between the groups, and sharing information about identities, it may be possible to determine how these communities may come together. For these discussions to take place, it is vital that correct information about asexuals be exchanged, in order to address some concerns about the 'threat' of the asexual community. This threat is based on both the societal assumption of sexual desire, something that is difficult to fix, and misconceptions about the asexual experience, which is easy to address with the proper information.

\section{Asexual Foundation: An Informative Future}

It should be clear by this point that asexual identity has been deeply challenged by both misinformation and a lack of concrete information regarding the orientation. There are still people who consider the label as part of mental or physical illness, and the orientation itself is multifaceted, with experiences differing between individuals. The argument about whether the orientation may be 'classified' in the LGBTQ+ community reveals deeper problems concerning the nature of minority status itself in terms of sexual orientation.
However, these problems do contain their own solution. If asexuals are suffering due to a lack of correct information, it follows that healing those wounds may come from further exploration of the orientation. Through further 1) academic discussion, 2) social education and 3) constructing a stronger community, these problems can be addressed.

\section{Academic Aces}

In terms of correcting misinformation, one powerful tool would be to conduct more studies on asexuality. Now that several studies have demonstrated the great likelihood that asexuality is an orientation in and of itself, others should follow. Some could focus on questions from the FAQ on AVEN; how romance and asexuality intersect, for example, or exploring the different 'levels' of asexuality. This type of exploration makes the orientation legitimate in the eyes of the academic community, which may lead to societal acceptance.

\section{Asexual Visibility}

The efforts to educate about asexuality must extend beyond the academic world. AVEN is an excellent source, but information must be more widely disseminated. This may be accomplished by efforts of asexuals themselves, either by sharing their own experiences both on and offline, discussing the different aspects of the orientation, and helping to address misconceptions and questions. However, it is not enough for the community to 
become more informed about themselves. In order to make asexuality visible, there must be efforts to reach outside, both to allosexual people and to people who may not realize they are asexual. By alerting society to create an understanding about the existence of asexuality as a real orientation it will be possible to confront the misinformation and marginalization of asexual people.

\section{Bridging the Divide in LGBTQ+}

These educational efforts will be a major component of bridging the divide between the asexual community and the LGBTQ+ community. If asexual people have their own vibrant, visible community, it is possible that they may not feel the need to become part of another community. On the other hand, with proper discussion and debate, it is possible that more LGBTQ+ groups will accept the reasoning of asexuality falling under the 'queer' spectrum, particularly for asexuals whose romantic orientations fall within this category. Without the 'threat' posed by inaccurate portrayals and understanding of asexuality, it is possible that asexuality will be embraced by the LGBTQ + community. Discussions create the potential for both communities to be able to understand each, and acknowledge their shared minority status.

\section{Conclusion}

Building an identity is never easy. There are multiple components, there is friction with established groups, and sometimes people will ascribe false assumptions to what makes that identity separate and valid. Asexuality faces all three of these challenges. While there has been progress in academic circles, there are still several false assumptions about what makes someone not experience attraction, or experience it at a lower level than others. These assumptions tear down the possibility that asexuality is, in and of itself, the reason for experiencing life in this matter. It threatens its status as a viable sexual orientation, a status which many argue is unfairly given. The range of asexual resources is also not very large which is problematic, as asexuality exists upon a spectrum with several variables, like sex drive and attraction levels, which are difficult to measure. Furthermore, its intersections with romantic orientations deepen the confusion, often blurring the line between asexual and sexual. While there are some sources, they are few in number and are not often easily found. Finally, asexuals face a great deal of confusion with regards to the LGBTQ+ community. While some argue that asexuality belongs in that community, other disagree, defining asexuality as a lack of orientation, which may even bring harm to the goals of the LGBTQ+ community. There is even confusion among asexuals themselves, as some feel that they are part of this community, while others are content to remain in the asexual community, or even to think of themselves as predominantly straight. 
As asexual experience has been stated over and over again, it is closely tied to selfidentifying. Research has changed the DSM to prove that identification is enough to preclude a diagnosis of HSDD. AVEN supports the right of anyone calling themselves asexual if they believe it applies, and there are several LGBTQ+ groups who accept asexual people if they define themselves as such. If this identity is to become widely accepted, the solution is more information. With academic research being done on the background and experience of asexuality, the orientation may be given social credence and will help to quash the false information and assumptions allosexual people often make about the 'reasons' behind someone identifying as asexual. This may lead to a higher visibility of asexuality in the public eye, creating more opportunities for people to discover this orientation and perhaps identify with it. New asexual identifying individuals may form connections through AVEN and other forums, perhaps creating more asexual sources for the curious, questioning person. It may even lead to more representation in popular media, which would allow for further attention to spark discussion within the community and society. Finally, the LGBTQ+ community and the asexual community need to understand that there is no threat to either group from the other's existence. It can be argued that by demonstrating an orientation that has little to do with sex of any kind, asexuals are proving that the default is not straight. Whether asexuals continue to build their own separate, vibrant community or LGBTQ+ gains an undisputed $A$, opening a discourse between these groups will lead to a healthy exchange of information between these minorities.

The idea of 'asexuality' is young in societal terms, and there are many questions that remain to be answered. With the proper application of research, understanding, and information sharing, it will become accepted.

\section{References}

AVEN [The Asexual Visibility and Education Network] (n.d.-a). General FAQ. Retrieved November 13, 2016, from http://www.asexuality.org/home/? $\mathrm{q}=$ general.html

AVEN [The Asexual Visibility and Education Network]. (n.d.-b). Overview. Retrieved November 13, 2016, from http://www.asexuality.org/home/? $\mathrm{q}=$ overview.html

Bogaert, Anthony F. (2006). Toward a conceptual understanding of asexuality. Review of General Psychology, 10(3).

Brotto, L. A., Yule, M. A., \& Gorzalka, B. B. (2015). Asexuality: an extreme variant of sexual desire disorder? Journal of Sexual Medicine, 12(3), 
646-660.

https://doi.org/10.1111/jsm.12806

Canning, Dominique A. (2015). Queering asexuality: Asexual-inclusion in queer spaces. McNair Scholars Research Journal, 8(1), Article 6.

Cerankowski, K. J., \& Milks, M. (2010). New Orientations: Asexuality and its implications for theory and practice. Feminist Studies, 36(3).

Decker, J.S. (2015). The invisible orientation: An introduction to asexuality. New York: Skyhorse Publishing.

Gressgård, R. (2013). Asexuality: From pathology to identity and beyond. Psychology \& Sexuality, 4(2), 179192.

https://doi.org/10.1080/19419899. 2013.774166
Halifax Public Libraries. (n.d.) Search keywords: asexuality, across library system. Included non-fiction and fiction results.

MacNeela, P., \& Murphy, A. (2015). Freedom, invisibility, and community: A qualitative study of self-identification with asexuality. Archives of Sexual Behavior, 44(3), 799-812.

Miller, S. (2015). A queer literacy framework promoting (a)gender and (a)sexuality self-determination and justice. English Journal, High School Edition, 104(5), 37-44.

Pinto, S. A. (2014). ASEXUally: On being an ally to the asexual community. Journal of $L G B T$ Issues in Counseling, 8(4), 331-343. https://doi.org/10.1080/15538605. 2014.960130 